The Astrophysical Journal, 249:592-601, 1981 October 15

(1) 1981. The American Astronomical Society. All rights reserved. Printed in U.S.A.

\title{
THE ABUNDANCES OF NEON, SULFUR, AND ARGON IN PLANETARY NEBULAE
}

\author{
S. C. BeCK, J. H. LACY, AND C. H. Townes \\ Department of Physics, University of California, Berkeley \\ L. H. AlLER \\ Astronomy Department, University of California, Los Angeles \\ T. R. Geballe \\ Mount Wilson and Las Campanas Observatories, Carnegie Institution of Washington \\ AND \\ F. BAAS \\ Sterrenwacht Huygens Laboratorium, Rijksuniversiteit te Leiden \\ Received 1980 November 10; accepted 1981 April 27
}

\begin{abstract}
Infrared observations of [Ne II], [S IV], and [Ar III] are used with optical observations to discuss the abundances of $\mathrm{Ne}, \mathrm{S}$, and $\mathrm{Ar}$ in 18 planetary nebulae. In addition, infrared observations of 18 other nebulae are presented. The derived abundances of $\mathrm{S}$ and Ar are each slightly enhanced relative to previous studies.
\end{abstract}

Subject headings: infrared: spectra - nebulae: abundances - nebulae: planetary

\section{INTRODUCTION}

A frequently encountered difficulty in determining the chemical composition of gaseous nebulae is that for most elements only a limited number of ionization stages are observed. Because of the severe deviations from thermodynamic equilibrium, extrapolation to allow for contributions of unobserved ionic stages can be troublesome.

For a few abundant elements, optical observations normally are entirely sufficient to determine the elemental abundance. Except in low excitation nebulae where neutral helium is important, $n(\mathrm{He}) / n(\mathrm{H})=\left[n\left(\mathrm{He}{ }^{+}\right)+\right.$ $\left.n\left(\mathrm{He}^{+2}\right)\right] / n\left(\mathrm{H}^{+}\right)$, so this abundance ratio is often easily established. In many nebulae, $n(\mathrm{O}) \approx n\left(\mathrm{O}^{+}\right)+n\left(\mathrm{O}^{+2}\right)$, so we can likewise obtain the $n(\mathrm{O}) / n(\mathrm{H})$ ratio with small corrections. Important ions of a few other elements are optically observable in some planetaries, e.g., $\mathrm{Cl}^{+}, \mathrm{Cl}^{+2}$, and $\mathrm{Cl}^{+3}$, and $\mathrm{Ar}^{+2}, \mathrm{Ar}^{+3}$, and $\mathrm{Ar}^{+4}$ in high excitation objects.

Some of the most heavily populated ionization stages of several important elements (notably $\mathrm{C}, \mathrm{N}, \mathrm{Ne}$, and $\mathrm{S}$ ), however, do not produce lines in the optical range. Carbon has no forbidden lines except the near infrared [C I] lines at $\lambda$ 9849.5, which are observable in a few objects that show either low excitation or a great range in excitation. For other ionization states of carbon, we must depend on ultraviolet radiation as observed by the IUE satellite or permitted transitions such as C III $\lambda 4267$ interpreted as recombination events. Lines of [N I] and [N II] are frequently observed, but in many nebulae most of the nitrogen must exist as $\mathrm{N}^{+2}$ or in yet higher ionization stages. The infrared [N III] 57.3 $\mu \mathrm{m}$ line is not observable from the earth's surface and is quite difficult to observe from the air. Here again, IUE observations are of great importance in assessing the nitrogen abundance since nitrogen is well represented by transitions in the $900-2000 \AA$ range.

The importance of infrared observations in determining abundances in planetary nebulae has been shown by many investigations; for a review see, e.g., Rank (1978). Among the medium mass abundant elements, infrared lines of neon, sulfur, and argon occur at $12.8 \mu \mathrm{m}, 10.5 \mu \mathrm{m}$, and $9.0 \mu \mathrm{m}$, respectively, all of which are accessible from the ground.

Neon is an example in point. In low excitation nebulae, much of the neon must exist as $\mathrm{Ne}^{+}$, which has no visible transitions. A frequent procedure (in the absence of observational infrared data) has been to estimate the total amount of neon by an equation suggested by Peimbert and Costero (1959):

$$
\frac{n(\mathrm{Ne})}{n(\mathrm{H})}=\frac{n\left(\mathrm{O}^{+}\right)+n\left(\mathrm{O}^{+2}\right)}{n\left(\mathrm{O}^{+2}\right)} \frac{n\left(\mathrm{Ne}^{+2}\right)}{n\left(\mathrm{H}^{+}\right)}
$$

but the validity of this expression has not been proven. If charge exchange is important for the reaction $\mathrm{O}^{+2}+\mathrm{H}^{0} \rightarrow$ $\mathrm{O}^{+}+\mathrm{H}^{+}$, but not for $\mathrm{Ne}^{+2}+\mathrm{H}^{0} \rightarrow \mathrm{Ne}^{+}+\mathrm{H}^{+}$, as recent calculations by Butler, Bender, and Dalgarno (1979) would 
suggest, the formula may be inadequate. Therefore, observations of the $12.8 \mu \mathrm{m}$ line of $\mathrm{Ne}$ II are crucial to accurate determinations of the $\mathrm{Ne}$ abundance in low excitation nebulae.

The problem for sulfur is somewhat different. The [S II] emission lines are often observed, but in all except the very lowest excitation objects, sulfur exists as ions of $\mathrm{S}^{+2}, \mathrm{~S}^{+3}$, and perhaps even higher stages. To employ Peimbert and Costero's (1969) extrapolation procedure, $n\left(\mathrm{~S}^{+}\right)+n\left(\mathrm{~S}^{+2}\right)$ are needed, as illustrated, for example, in sulfur abundance determinations for NGC 6720 (Aller 1976), and more recently in determinations of sulfur abundances in other planetaries (Barker 1978; Aller 1978 $a, b$ ). It is best to use the nebular [S III] $\lambda 9069$ because $\lambda 9536$ tends to be confused with Paschen 8 at the resolutions normally used. A number of observers have measured these near-infrared lines, e.g., O'Dell (1962), Liller and Aller (1963), Perinotto (1974), and Barker (1978); a compilation of data available to 1975 is given by Kaler (1976). An alternate procedure is to use the [S III] auroral $\lambda 6312$ transition to estimate the $\mathrm{S}^{+2}$ concentration. The result is more sensitive to $T_{e}$, but the advantage is that $\lambda 6312$ is usually observed in the same areas of the nebula as other visual region lines are observed, whereas observations of [S III] nebular transitions usually involve integration over the entire nebula. Simple considerations of the ionization structure show that in all planetaries of medium to high excitation, sulfur exists mostly as $\mathbf{S}^{+3}$ or perhaps in even higher stages of ionization. Other than far ultraviolet lines which have not yet been observed, the $10.5 \mu \mathrm{m}$ line of [S IV] is the only direct link to this ionization stage available. Even when $\left[n\left(\mathrm{~S}^{+}\right)+n\left(\mathrm{~S}^{+2}\right)\right]$ has been determined seemingly reliably, extrapolation for higher ionization stages is difficult, and it is desirable to have an independent check.

We do not add an additional ionization stage of argon by observing the $9.0 \mu \mathrm{m}$ [Ar III] line; rather, we get corroborative evidence on the concentration of $\mathrm{Ar}^{+2}$. Since we can also estimate $n\left(\mathrm{Ar}^{+2}\right)$ from $\lambda 7135$, a comparison of the two determinations will give clues to possible errors in the assumed value of $T_{e}$, the influence of interstellar extinction or differential internal extinction in the nebula itself, and cast some light, perhaps, on the basic atomic data.

In the present investigation, new infrared observations of [Ne II], [Ar III], and [S IV], together with optical observations of other ionization states of these elements, are used to discuss the abundances of neon, argon, and sulfur in 18 planetary nebulae. In addition, one or more of the infrared lines were detected in 18 other nebulae and are reported here. [S IV] was detected in approximately $90 \%$ of the objects observed, [ $\mathrm{Ar} \mathrm{III}]$ in about $80 \%$, and $[\mathrm{Ne} \mathrm{II}]$ in roughly one-third.

It should be noted that optical observations typically include only a limited region of the nebula, while the infrared measurements frequently involve integration over the entire nebular image. Hence, except for small or rather uniformly excited nebulae, comparison of slit spectroscopy results or ITS data with integrated measurements obtained with photoelectric scanners, photometers, infrared detectors, or the IUE must be made cautiously. In particular, highly structured objects such as NGC 7009 present substantial difficulties (see Czyzak and Aller 1979).

\section{OBSERVATIONS}

The infrared observations were made during 1976-1979 with the Lick Observatory $3 \mathrm{~m}$ Shane telescope and with the Las Campanas Observatory $2.5 \mathrm{~m}$ duPont telescope, using a liquid nitrogen cooled, piezoelectrically scanned Fabry-Perot interferometer in tandem with a liquid helium cooled grating monochromator (Lacy 1979). The beam size was 6" (FWHM) for the northern objects and 7" for the southern objects. Chopping was employed using beam separations of typically $1^{\prime}$. Flux calibration was achieved by chopping between an ambient temperature blackbody and the sky, since accurate narrow-band mid-infrared stellar fluxes are not known. The calibration measurements were done several times per night and are consistent with each other to about $\pm 10 \%$. There is the possibility of further systematic errors in the flux calibration if the temperature of the sky or the blackbody is not well known; therefore, the total uncertainty in the flux calibration is estimated to be $\pm 15 \%$. This uncertainty is independent of line flux level. Usually lines from one or more sources measured during one observing period were reobserved during the next to assure the consistency of the flux measurements. Infrared line fluxes reported in Table 1 are taken from a computer generated Lorentzian best fit to the observed line, in order to minimize possible bias in the analysis.

Most of the optical intensities used for ionic concentration calculations were measured with the image tube scanner at the Lick Observatory $3 \mathrm{~m}$ telescope and calibrated by photoelectric photometry (Aller and Czyzak 1979). Typical aperture sizes were $2^{\prime \prime} \times 4^{\prime \prime}$. For six of the planetaries, observations of [S III] $(\lambda 9069)$ were obtained in October 1979 with the Lick $3 \mathrm{~m}$ telescope and UCLA Reticon system. Errors in line intensities should be equal to or less than about $15 \%$ except for very weak lines.

The total fluxes of [Ne II] $(12.8 \mu \mathrm{m})$, [Ar III] $(9.0 \mu \mathrm{m})$, and [S IV] $(10.5 \mu \mathrm{m})$ are given in columns (2)-(4) of Table 1 . The notation ... indicates that the line was not observed. A dash indicates that the line was not detected; upper limits are $\sim 10^{-12} \mathrm{erg} \mathrm{s}^{-1} \mathrm{~cm}^{-2}$ in the 6" or 7" diameter aperture. In addition, Ar III ( $\left.\lambda 7135\right)$, the recent S III ( $\lambda$ 9069), and the $\mathrm{H} \beta$ total fluxes, all corrected for interstellar extinction, are given for comparison in columns (5)-(7) of Table 1 . Wherever possible, the $\mathrm{H} \beta$ fluxes are predicted from $5 \mathrm{GHz}$ continuum fluxes (Milne and Aller 1975, eq. 6). 
TABLE 1

TOTAL LINE FLUXES ${ }^{\mathrm{a}}$

\begin{tabular}{|c|c|c|c|c|c|c|c|}
\hline \multirow[b]{2}{*}{$\begin{array}{l}\text { NeBULA } \\
(1)\end{array}$} & \multicolumn{6}{|c|}{$F\left(10^{-11} \mathrm{erg} \mathrm{s}^{-1} \mathrm{~cm}^{-2}\right)$} & \multirow[b]{2}{*}{$\begin{array}{l}\text { COMMENTS } \\
(8)\end{array}$} \\
\hline & $\begin{array}{c}\text { Ne II } \\
12.8 \mu \mathrm{m} \\
(2)\end{array}$ & $\begin{array}{c}\text { S IV } \\
10.5 \mu \mathrm{m} \\
(3)\end{array}$ & $\begin{array}{c}\text { Ar III } \\
9.0 \mu \mathrm{m} \\
(4)\end{array}$ & $\begin{array}{c}\mathrm{Ar} \mathrm{III}^{\mathrm{b}} \\
7135 \AA \\
(5)\end{array}$ & $\begin{array}{c}\mathrm{S} \mathrm{III}^{\mathrm{c}} \\
9069 \AA \\
(6)\end{array}$ & $\begin{array}{c}\mathrm{H}_{\beta}{ }^{\mathrm{d}} \\
4861 \AA \\
(7)\end{array}$ & \\
\hline IC 418 & 26.4 & n.d. ${ }^{h}$ & 1.0 & $2.6(1.22)$ & 10.6 & 49. $(0.22)$ & $\left\{\begin{array}{l}\text { Ne II mapped } \\
\text { Ar III(9.0): see notes e, g. }\end{array}\right.$ \\
\hline IC 2165 & n.d. ${ }^{h}$ & 1.3 & n.d. ${ }^{h}$ & $0.27(1.51)$ & 0.43 & $3.9(0.46)$ & \\
\hline NGC 2440 & n.d. ${ }^{h}$ & n.d. ${ }^{h}$ & see comment & $2.1(1.82)$ & $\ldots$ & $9.3(0.42)$ & Ar III(9.0): $0.156^{\prime \prime} \mathrm{NW}$ of star. \\
\hline NGC 2792 & n.d. ${ }^{\mathrm{h}}$ & 1.8 & n.d. ${ }^{h}$ & $\ldots$ & $\ldots$ & $2.0(0.53)$ & ... \\
\hline NGC 2867 & n.d. ${ }^{\mathrm{h}}$ & 1.8 & see comment & $1.09(1.50)$ & $\ldots$ & $7.4(0.44)$ & Ar III(9.0): 0.24 on star, $0.285^{\prime \prime} \mathrm{E}$ \\
\hline IC 2501 & 1.8 & 3.6 & 1.3 & $\ldots$ & $\ldots$ & $7.4(0.52)$ & $\ldots$ \\
\hline NGC 3242 & n.d. ${ }^{\mathrm{h}}$ & 8.3 & 0.6 & $1.8(1.17)$ & $\ldots$ & $25.7(0.18)$ & S IV and $\operatorname{Ar} \operatorname{III}(9.0)$ mapped in inner (brighter) ring. \\
\hline NGC 2621 & n.d. ${ }^{\mathrm{h}}$ & 4.5 & 1.3 & $\ldots$ & $\ldots$ & $5.5(0.93)$ & $\ldots$ \\
\hline NGC 3918 & 0.6 & 11.0 & 1.0 & $\ldots$ & $\ldots$ & 22. $(0.33)$ & $\ldots$ \\
\hline IC 3568 & n.d. ${ }^{h}$ & 0.3 & n.d. ${ }^{\mathrm{h}}$ & $\ldots$ & $\ldots$ & $1.5(0.00)$ & $\ldots$ \\
\hline $\mathrm{MyCn} 18$ & 1.8 & 0.7 & 1.7 & $\ldots$ & $\ldots$ & $2.8(0.74)$ & $=\mathrm{He} 2-95=\mathrm{RCW} 77$ \\
\hline NGC 5882 & n.d. ${ }^{\mathrm{h}}$ & 10.4 & 0.7 & $\cdots$ & $\ldots$ & $10.5(0.42)$ & 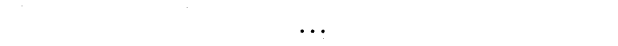 \\
\hline IC 4406 & 0.17 & $\ldots$ & $\ldots$ & $\ldots$ & $\ldots$ & $3.5(0.27)$ & $\ldots$ \\
\hline $\mathrm{He} 2-131$ & 7.0 & $\ldots$ & $\ldots$ & $\ldots$ & $\ldots$ & $9.1(0.21)$ & $=$ My 90 \\
\hline IC 4593 & n.d. ${ }^{h}$ & 1.0 & 0.4 & $0.25(1.0)$ & $\ldots$ & $2.9(0.01)$ & S IV and $\operatorname{Ar} \operatorname{III}(9.0)$ : see note $f$. \\
\hline NGC 6210 & n.d. ${ }^{h}$ & 16.3 & 0.8 & $1.0(1.0)$ & $\ldots$ & $10.5(0.08)$ & S IV: see note e; $\operatorname{Ar}$ ill(9.0) see notes $f, g$. \\
\hline IC 4634 & 0.2 & $\ldots$ & & $\ldots$ & $\ldots$ & $3.7(0.49)$ & $\ldots$ \\
\hline Me 2-1 & n.d. ${ }^{\mathrm{h}}$ & 0.7 & n.d. ${ }^{\mathrm{h}}$ & $0.08(1.25)$ & $\ldots$ & $0.79(0.22)$ & $=\mathrm{He} 2-126=\mathrm{VV} 124$ \\
\hline NGC 6339ctr & n.d. ${ }^{\mathrm{h}}$ & n.d. ${ }^{\mathrm{h}}$ & 0.3 & $\ldots$ & $\ldots$ & $\ldots$ & 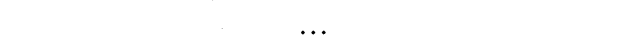 \\
\hline $10^{\prime \prime} \mathrm{N}$ & n.d. ${ }^{\mathrm{h}}$ & 4.2 & 1.2 & $\ldots$ & $\ldots$ & $\ldots$ & $\ldots$ \\
\hline $\mathrm{Hb} 4$ & n.d. ${ }^{\mathrm{h}}$ & 8.8 & 3.0 & $\ldots$ & $\ldots$ & $5.1(1.49)$ & $=\mathrm{VV} 232$ \\
\hline $\mathrm{Hb} 6$ & n.d. ${ }^{h}$ & 11.4 & 4.2 & $\ldots$ & $\ldots$ & $6.6(1.57)$ & $=\mathrm{VV} 285$ \\
\hline NGC 6537 & 2.1 & 13.3 & 2.3 & $\ldots$ & $\ldots$ & $12.0(1.79)$ & (n) \\
\hline NGC 6572 & 2.1 & 11.2 & 4.7 & $8.4(1.38)$ & $\ldots$ & 41 & $\left\{\begin{array}{l}\text { Ne II and } \mathrm{Ar} \text { III }(9.0): \text { see note } \mathrm{f} \\
\text { S IV: see note e. }\end{array}\right.$ \\
\hline NGC 6741 & 1.1 & 1.7 & 0.9 & $1.36(3.0)$ & $\ldots$ & $5.5(1.23)$ & \\
\hline NGC 6790 & n.d. ${ }^{h}$ & 0.35 & 0.5 & $0.35(1.73)$ & $\ldots$ & $7.1(0.60)$ & $\ldots$ \\
\hline NGC 6803 & 1.3 & 2.2 & 0.9 & $\ldots$ & $\ldots$ & $3.6(0.71)$ & $\ldots$ \\
\hline BD $30^{\circ} 3639$ & 3.8 & n.d. ${ }^{\text {h }}$ & 0.5 & $\ldots$ & $\ldots$ & $16.2(0.22)$ & $\ldots$ \\
\hline NGC 6884 & n.d. ${ }^{h}$ & 7.4 & 0.9 & $1.79(2.67)$ & $\ldots$ & $9.5(1.09)$ & $\ldots$ \\
\hline NGC 6886 & n.d. ${ }^{h}$ & 4.2 & n.d. ${ }^{h}$ & $0.45(2.23)$ & $\ldots$ & $2.4(0.89)$ & $\ldots$ \\
\hline IC 4997 & n.d. ${ }^{h}$ & 2.0 & 0.2 & $1.63(1.12)$ & $\ldots$ & $4.4(0.13)$ & $\ldots$ \\
\hline NGC 7009 & n.d. ${ }^{h}$ & 22.4 & 4.2 & $2.96(1.08)$ & $\ldots$ & 23. $(0.14)$ & $\left\{\begin{array}{l}\text { Ar III(9.0): } 1.8 \text { in center, } 1.26^{\prime \prime} \mathrm{E} ; \mathrm{S} \text { IV: } 8.4 \text { in } \\
\text { center, } 7.07^{\prime \prime} \text { E bilateral symmetry assumed. }\end{array}\right.$ \\
\hline NGC 7026 & n.d. ${ }^{h}$ & 19.2 & 1.4 & $1.82(2.05)$ & 4.0 & $7.9(0.80)$ & $\left\{\begin{array}{l}\text { S Iv: } 9.0 \text { in } \mathrm{E} \text { knot, } 10.2 \text { in W; } \\
\text { flux assumed concentrated in knots. }\end{array}\right.$ \\
\hline $\begin{array}{l}\text { NGC } 7027 \\
\text { bright }\end{array}$ & & & & $40.5(3.42)$ & $\cdots$ & 178. (1.37) & ... \\
\hline spot & 3.57 & 19.3 & 3.66 & $\ldots$ & $\ldots$ & $\ldots$ & $\ldots$ \\
\hline $3^{\prime \prime} \mathrm{S}, 2^{\prime \prime} \mathrm{E}$ & 3.24 & 18.9 & 3.67 & $\ldots$ & $\ldots$ & $\ldots$ & $\cdots$ \\
\hline $4^{\prime \prime} \mathrm{E}$ & $\ldots$ & 7.95 & 2.89 & $\ldots$ & $\ldots$ & $\ldots$ & $\ldots$ \\
\hline $5^{\prime \prime} \mathrm{S}, 2^{\prime \prime} \mathrm{W}$ & $\ldots$ & 4.85 & & $\ldots$ & $\ldots$ & $\ldots$ & $\ldots$ \\
\hline $2^{\prime \prime} \mathrm{S}, 2^{\prime \prime} \mathrm{W}$ & $\ldots$ & $\ldots$ & 0.96 & $\ldots$ & $\ldots$ & $\ldots$ & $\ldots$ \\
\hline IC 5117 & n.d. ${ }^{h}$ & 2.8 & 0.4 & $0.56(3.22)$ & 0.74 & $3.9(1.30)$ & $\ldots$ \\
\hline IC 5217 & n.d. ${ }^{\mathrm{h}}$ & 2.0 & $\ldots$ & $0.18(1.5)$ & 0.44 & $1.86(0.45)$ & $\ldots$ \\
\hline NGC 7662 & n.d. ${ }^{b}$ & 6.7 & $\ldots$ & $1.43(1.15)$ & 1.36 & $15.1(0.16)$ & $\left\{\begin{array}{l}\text { S Iv: } 2.3 \text { in center, } 2.46^{\prime \prime} \mathrm{NE}, 2.06^{\prime \prime} \mathrm{NW} \text {; all flux } \\
\text { assumed observed. }\end{array}\right.$ \\
\hline
\end{tabular}

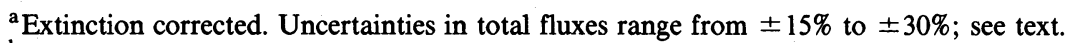

${ }^{b}$ Extinction correction factors, in parentheses, taken from Kaler 1976.

${ }^{\mathrm{c}}$ Extinction correction obtained from nearby Paschen series lines and the calculated line intensity ratios of Brocklehurst (1971).

dFrom Milne and Aller 1975 or O'Dell 1962; $C$-values, in parentheses, from O'Dell and Terzian 1970, Milne and Aller 1975, Kaler 1976, Aller and Czyzak 1979, and Aller, previously unpublished.

e Detected only in central one of several positions observed; central position assumed to contain all flux.

${ }^{\text {f }}$ Only central position observed; assumed to contain all flux.

g Probably underestimates total flux.

${ }^{\mathrm{h}}$ Not detected; $<0.1 \times 10^{-11} \mathrm{erg} \mathrm{s}^{-1} \mathrm{~cm}^{-2}$ in $6^{\prime \prime}$ or $7^{\prime \prime}$ diameter aperture. 
Of the 18 planetaries modeled in this paper, eight were larger than the infrared beam. IC 418 was mapped completely in Ne II, and the inner ring of NGC 3242 was mapped in all three ions. The remaining extended planetaries were not completely mapped, and it was necessary to assume intensity distributions to calculate total fluxes. These assumptions were based on the infrared intensities, the appearance of the optical images, and the known tendency for the ions of high excitation, such as S IV, to be more centrally concentrated than those of low excitation, such as Ne II. The uncertainty in scaling the measured infrared line fluxes may be as large as $\pm 25 \%$ in some cases. Therefore the uncertainties in most total infrared line fluxes in Table 1 are thought to range from $\pm 15 \%$ for the smaller nebulae to $\pm 30 \%$ for the larger nebulae. There are some cases (see below) where this uncertainty may be greater than $\pm 30 \%$. Errors in scaling optical and near-infrared line fluxes are thought to be $\pm 15 \%$ for most lines, making the uncertainty in those total fluxes typically $\pm 20 \%$. Details regarding the infrared flux calculations are given in the comments in Table 1 . Nebulae with no comments have optical images smaller than the infrared beam.

The present infrared observations are the most extensive study to date of these fine-structure lines in planetary nebulae. Previous observations include those of Gillett, Merrill, and Stein (1972), Grasdalen (1979), Aitken et al. (1979), and Dinerstein (1980). Eighteen of the planetaries discussed in this paper were observed by one or more of those authors. A comparison of the present and previous observations is given in Table 2.

The most significant discrepancies between the results of this survey and those of other infrared workers are in the S IV fluxes. For IC 3568 and NGC 6790 the present measurements are as much as an order of magnitude lower than those reported by other observers. In addition, the S IV flux for NGC 6803 is 2.6 times lower than previously reported. For NGC 6537, however, the S IV flux is 3.3 times higher than the previously reported value, and in several other cases (NGC 6210, NGC 6889, NGC 6886, NGC 7026, and IC 4997) it is up to twice that found by other workers.

TABLE 2

COMPARISON OF INFRARED MEASUREMENTS ${ }^{\mathrm{a}}$

\begin{tabular}{|c|c|c|c|c|c|c|}
\hline & This Paper & $\left(6^{\prime \prime}\right.$ or $\left.7^{\prime \prime}\right)$ & Aitken et al. & Grasdalen $\left(11^{\prime \prime}\right)$ & $\begin{array}{l}\text { Gillett, Merrill, and } \\
\text { Stein }\left(11^{\prime \prime}\right)\end{array}$ & Dinerstein $\left(10^{\prime \prime}\right)$ \\
\hline IC 3568 & S IV & 0.3 & $\ldots$ & $\ldots$ & $\ldots$ & 2.2 \\
\hline IC $4593 \ldots \ldots \ldots$ & S IV & 1.0 & $\ldots$ & $\ldots$ & 1.4 & 1.4 \\
\hline \multirow[t]{2}{*}{ N $6210 \ldots \ldots \ldots$} & S IV & 16.3 & $\ldots$ & $11.5 \pm 0.5$ & 8 & 8.4 \\
\hline & Ar III & 0.8 & $\cdots$ & $2.8 \pm 1.0$ & $\ldots$ & $\ldots$ \\
\hline N $6339 \ldots \ldots \ldots$ & S IV & $>4.2$ & $\ldots$ & $\ldots$ & 3 & $\ldots$ \\
\hline N $6537 \ldots \ldots \ldots$ & S IV & 13.3 & $\ldots$ & $\ldots$ & 4 & $\ldots$ \\
\hline \multirow[t]{2}{*}{ N $6572 \ldots \ldots \ldots$} & S IV & 11.2 & $\ldots$ & $7.8 \pm 1.1$ & 9 & 7.2 \\
\hline & Ar III & 4.7 & $\ldots$ & $2.8 \pm 1.0$ & $\ldots$ & $\ldots$ \\
\hline \multirow[t]{3}{*}{ N $6741 \ldots \ldots \ldots$} & $\mathrm{Ne}$ II & 1.1 & $\begin{array}{l}\cdots \\
\cdots\end{array}$ & $0.9 \pm 0.5$ & $\cdots$ & 1.0 \\
\hline & S IV & 1.7 & $\ldots$ & $2.5 \pm 0.5$ & $\ldots$ & $\ldots$ \\
\hline & $\mathrm{Ar}$ III & 0.9 & $\ldots$ & $1.33 \pm 0.3$ & $\ldots$ & $\ldots$ \\
\hline \multirow{3}{*}{ N 6790} & $\mathrm{Ne}$ II & $<0.1$ & $<0.5$ & $\ldots$ & $\ldots$ & $\ldots$ \\
\hline & S IV & 0.35 & $0.98 \pm 0.10(3 . " 4)$ & $\ldots$ & 1.4 & 3.6 \\
\hline & Ar III & 0.5 & $0.30 \pm 0.25$ & $\ldots$ & $\ldots$ & $\ldots$ \\
\hline \multirow{2}{*}{ N $6803 \ldots \ldots \ldots$} & S IV & 2.2 & $\ldots$ & $5.7 \pm 0.6$ & $\ldots$ & $\ldots$ \\
\hline & Ar III & 0.9 & $\ldots$ & $1.0 \pm 0.33$ & $\ldots$ & $\ldots$ \\
\hline \multirow[t]{2}{*}{ N $6884 \ldots \ldots \ldots$} & S IV & 7.4 & $\cdots$ & $3.5 \pm 0.3$ & 6 & 4.0 \\
\hline & Ar III & 0.9 & $\ldots$ & $0.60 \pm 0.22$ & $\ldots$ & $\ldots$ \\
\hline N 6886 & S IV & 4.2 & $\ldots$ & $1.7 \pm 0.4$ & $\ldots$ & $\ldots$ \\
\hline \multirow[t]{2}{*}{ IC 4997} & S IV & 2.0 & $\ldots$ & $0.97 \pm 0.23$ & 3 & 0.8 \\
\hline & Ar III & 0.2 & $\ldots$ & $0.57 \pm 0.29$ & & $\ldots$ \\
\hline N $7009 \ldots \ldots \ldots$ & S IV & 22.4 & $\ldots$ & $\ldots$ & 16 & $\ldots$ \\
\hline \multirow[t]{2}{*}{ N $7026 \ldots \ldots \ldots$} & S IV & 19.2 & $\ldots$ & $10.3 \pm 0.6$ & 9 & $\ldots$ \\
\hline & Ar III & 1.4 & $\ldots$ & 1.2 & $\ldots$ & $\ldots$ \\
\hline IC 5117 & S IV & 2.8 & $\ldots$ & $2.6 \pm 0.5$ & $\ldots$ & $\ldots$ \\
\hline IC $5217 \ldots \ldots \ldots$ & S IV & 2.0 & $\ldots$ & $\ldots$ & 2 & $\ldots$ \\
\hline $\mathrm{N} 7662 \ldots \ldots \ldots$ & S IV & 6.7 & $\ldots$ & $\ldots$ & 9 & $\ldots$ \\
\hline \multirow[t]{3}{*}{ N 7027} & $\mathrm{Ne}$ & $>6$ & $19.7 \pm 4.0$ & $\ldots$ & $\ldots$ & $\ldots$ \\
\hline & S IV & $\gtrsim 40$ & $48.8 \pm 2.7\left(20^{\prime \prime}\right)$ & $\ldots$ & $\ldots$ & $\ldots$ \\
\hline & Ar III & $\gtrsim 7$ & $12.8 \pm 2.6$ & $\ldots$ & $\ldots$ & $\ldots$ \\
\hline
\end{tabular}

${ }^{\text {a} A l l ~ f l u x e s ~ a r e ~ i n ~ u n i t s ~ o f ~} 10^{-11} \mathrm{erg} \mathrm{s}^{-1} \mathrm{~cm}^{-2}$. Uncertainties are $\pm 30 \%$ for the measurements of Gillett, Merrill, and Stein, and $\pm 20 \%$ for the measurements of Dinerstein. Uncertainties are $\pm 15 \%$ in the present work for those nebulae which are smaller than the beam; for the larger nebulae, the uncertainty may be as large as $\pm 30 \%$. 
BECK ET AL.

TABLE 3

IONIC CONCENTRATIONS

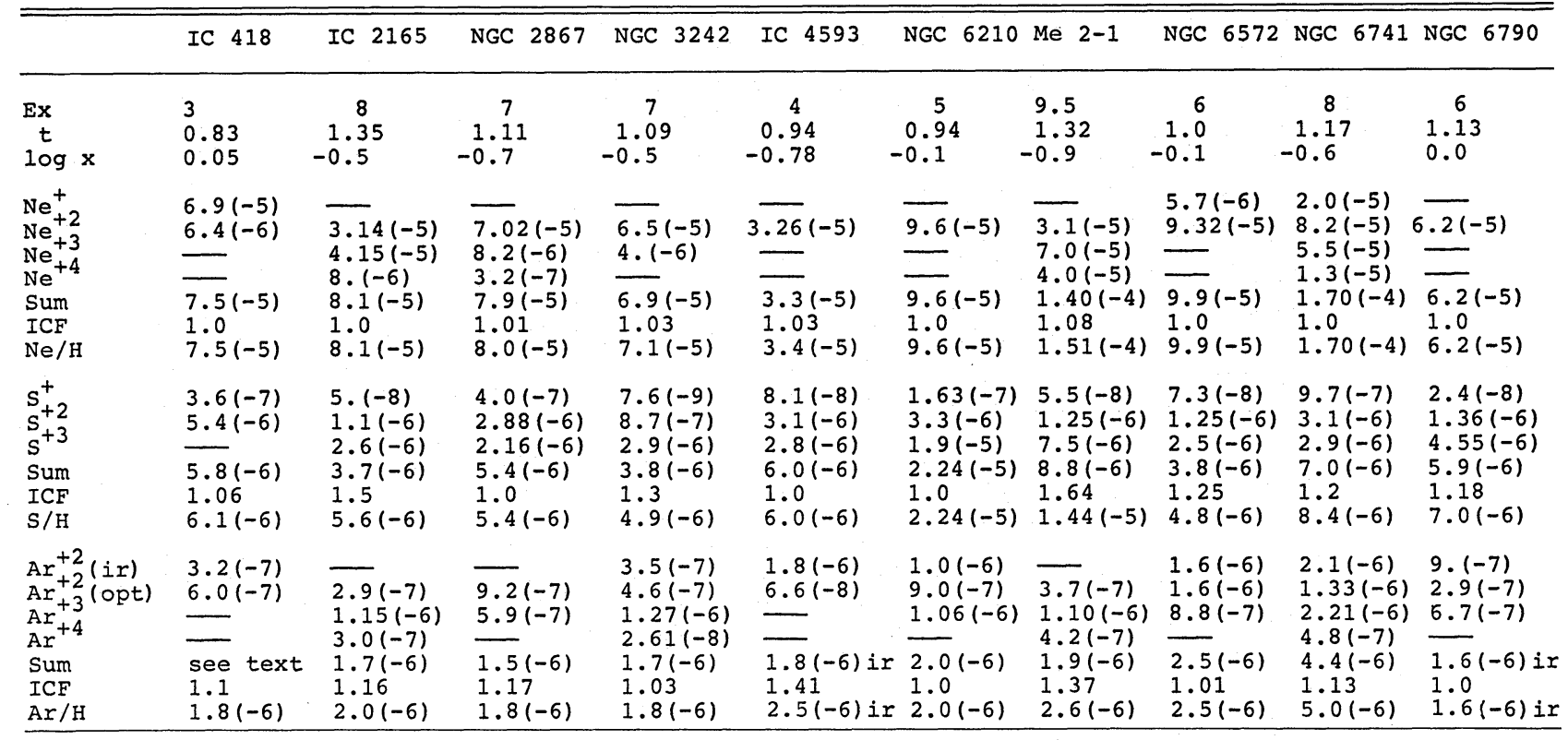

The larger fluxes from NGC 6210 and NGC 7026 reported here may result from more complete maps. The approximate factor of 2 differences for the other nebulae mentioned above likely are largely due to differences in beam sizes and in pointing. Many planetary nebulae are known to be stratified, so that attempts to correct for incomplete coverage of these objects may lead to erroneous results. The large discrepancies in IC 3568 and NGC 6790, however, cannot be explained on either of the above grounds. Because the result of Dinerstein (1980) for S IV in NGC 6790 appears most in accord with the other measured fluxes and known excitation of this nebula, it is used to derive the results in Tables 3 and 4.

\section{ANALYSIS}

\section{a) Use of Nebular Models}

Although observations of as many ionic stages as possible for every element are desirable, it often happens that all of the relavent ionization stages are not or cannot be covered. Therefore, even under the most favorable situations, it is often necessary to obtain ionization correction factors (ICFs). Although the empirical procedures previously described are popular and may be practical for $\mathrm{N}, \mathrm{O}, \mathrm{Ne}$, and sometimes $\mathrm{S}$ in nebulae of low to medium excitation (see, e.g., Grandi and Hawley 1978), the use of nebular models to derive ICFs is to be preferred for a number of reasons. It is often possible to represent intensities of the principal lines to within observational errors. Further detailed physical processes, such as charge exchange, can be considered. Even when a detailed representation of line intensities is not possible, the model can reproduce the general level of ionization and thus serve as an interpolation device.

Our approach has been to calculate models that reproduce the line intensities as closely as possible for most of the nebulae. Because of the expense involved, it was not possible to calculate detailed models of every nebula, however. Therefore, a different procedure was employed: computation of a network of models. By interpolation in this network, it was usually possible to reproduce the level of ionization and excitation appropriate to any individual object.

The model calculation procedures and underlying assumptions are described by Keyes and Aller (1978). The newer models employed in the present analysis involve improved rate coefficients for atomic processes and refined integration procedures. For a given density, $n_{\mathrm{H}}$, the general character of the emitted spectrum is determined by the stellar radius, $R^{*}$, and flux distribution $F^{*}(\nu)$, the chemical composition of the gas, and the truncation radius, $r_{c}$, of the nebular shell, or more specifically, the total amount of material in the shell. One can also introduce a variable density distribution $n_{\mathrm{H}}(r)$ or partial shells of differing density.

The overwhelming abundances of $\mathrm{H}$ and $\mathrm{He}$ strongly affect the radiation field within the nebula. The Strömgren radius, $r_{\mathrm{S}}$, at which hydrogen becomes neutral, fixes the nebular radius when the total mass of the surrounding shell is 
TABLE 3-Continued

\begin{tabular}{|c|c|c|c|c|c|c|c|c|}
\hline & NGC 6803 & NGC 6884 & NGC 6886 & NGC 7009 & NGC 7026 & IC 5117 & IC 5217 & NGC 7662 \\
\hline $\begin{array}{l}E x \\
t \\
\log x\end{array}$ & $\begin{array}{r}5 \\
0.93 \\
-0.05\end{array}$ & $\begin{array}{r}6 \\
1.05 \\
-0.05\end{array}$ & $\begin{array}{r}8 \\
1.27 \\
-0.20\end{array}$ & $\begin{array}{r}6 \\
0.95 \\
-0.14\end{array}$ & $\begin{array}{r}0.9 \\
-0.1 \\
-0.1\end{array}$ & $\begin{array}{l}1.18 \\
0.5\end{array}$ & $\begin{array}{c}1.1^{6} \\
-0.1\end{array}$ & $\begin{array}{c}1.3^{8} \\
-0.4^{8}\end{array}$ \\
\hline $\begin{array}{l}\mathrm{Ne}^{+} \\
\mathrm{Ne}^{+2} \\
\mathrm{Ne}^{+3} \\
\mathrm{Ne}^{+4} \\
\mathrm{Sum} \\
\mathrm{ICF} \\
\mathrm{Ne} / \mathrm{H}\end{array}$ & $\begin{array}{l}4.5(-5) \\
1.93(-4) \\
\overline{2} \\
2.38(-4) \\
1.0 \\
2.38(-4)\end{array}$ & $\begin{array}{l}\overline{8.2}(-5) \\
1.0(-5) \\
\overline{9.2}(-5) \\
1.03 \\
9.5(-5)\end{array}$ & $\begin{array}{l}\overline{6.0}(-5) \\
7.1(-5) \\
1.9(-5) \\
1.5(-4) \\
1.08 \\
1.62(-4)\end{array}$ & $\begin{array}{l}\bar{T} \\
1.45(-4) \\
3 .(-6) \\
1.48(-4) \\
1.02 \\
1.50(-4)\end{array}$ & $\begin{array}{l}\bar{T} \\
\frac{1.62}{1.62}(-4) \\
1.13 \\
1.83(-4)\end{array}$ & $\begin{array}{l}\overline{8.2}(-5) \\
9.6(-6) \\
\overline{9.2}(-5) \\
1.02 \\
9.4(-5)\end{array}$ & $\begin{array}{l}\overline{7.2}(-5) \\
\overline{7.2(}(-5) \\
1.16 \\
8.4(-5)\end{array}$ & $\begin{array}{l}\overline{3.4}(-5) \\
3.07(-5) \\
1.8(-6) \\
6.6(-5) \\
1.0 \\
6.6(-5)\end{array}$ \\
\hline $\begin{array}{l}S^{+} \\
S^{+2} \\
S^{+3} \\
\text { Sum } \\
I C F \\
S / H\end{array}$ & $\begin{array}{l}5.4(-7) \\
6.3(-6) \\
6.4(-6) \\
1.32(-5) \\
1.0 \\
1.32(-5)\end{array}$ & $\begin{array}{l}8.0(-8) \\
2.2(-6) \\
7.4(-6) \\
9.7(-6) \\
1.15 \\
1.11(-5)\end{array}$ & $\begin{array}{l}1.75(-7) \\
2.24(-6) \\
1.48(-5) \\
1.72(-5) \\
1.0 \\
1.72(-5)\end{array}$ & $\begin{array}{l}2.0(-7) \\
5.0(-6) \\
7.5(-6) \\
1.27(-5) \\
1.15 \\
1.46(-5)\end{array}$ & $\begin{array}{l}8.4(-7) \\
1.08(-5) \\
2.60(-5) \\
3.76(-5) \\
1.10 \\
4.1(-5)\end{array}$ & $\begin{array}{l}8.2(-8) \\
2.44(-6) \\
7.8(-6) \\
1.03(-5) \\
1.18 \\
1.21(-5)\end{array}$ & $\begin{array}{l}1.1(-7) \\
3.5(-6) \\
9.9(-6) \\
1.35(-5) \\
1.28 \\
1.73(-5)\end{array}$ & $\begin{array}{l}1.4(-8) \\
1.10(-6) \\
3.65(-6) \\
4.7(-6) \\
1.43 \\
6.7(-6)\end{array}$ \\
\hline $\begin{array}{l}\mathrm{Ar}^{+2} \text { (ir) } \\
\mathrm{Ar}_{+2} \text { (opt) } \\
\mathrm{Ar}_{+3} \text { (opt } \\
\mathrm{Ar}^{+4} \\
\mathrm{Sum} \\
\mathrm{ICF} \\
\mathrm{Ar} / \mathrm{H}\end{array}$ & $\begin{array}{l}\frac{4.04}{1.74}(-6) \\
\frac{5.8}{5.8}(-6) \\
1.0 \\
5.8(-6)\end{array}$ & $\begin{array}{l}1.34(-6) \\
1.34(-6) \\
2.26(-6) \\
1.01(-7) \\
3.7(-6) \\
1.03 \\
3.8(-6)\end{array}$ & $\begin{array}{l}\overline{8.9}(-7) \\
1.06(-6) \\
4.0(-7) \\
2.35(-6) \\
1.2 \\
2.8(-6)\end{array}$ & $\begin{array}{l}2.1(-6) \\
1.6(-6) \\
1.9(-6) \\
3.7(-7) \\
4.4(-6) \\
1.0 \\
4.4(-6)\end{array}$ & $\begin{array}{l}2.8(-6) \\
2.3(-6) \\
1.61(-6) \\
4.1(-6) \\
1.06 \\
4.4(-6)\end{array}$ & $\begin{array}{l}1.35(-6) \\
0.8(-6) \\
1.20(-6) \\
3.8(-8) \\
2.3(-6) \\
1.02 \\
2.4(-6)\end{array}$ & $\begin{array}{l}\overline{6.3}(-7) \\
1.42(-6) \\
\overline{2.05}(-6) \\
1.10 \\
2.3(-6)\end{array}$ & $\begin{array}{l}\overline{4.4}(-7) \\
1.41(-6) \\
1.5(-7) \\
2.0(-6) \\
1.05 \\
2.1(-6)\end{array}$ \\
\hline
\end{tabular}

IC 418: Several theoretical models have been calculated for this object, e.g., Flower 1969, Buerger 1973, Aller et al. 1980. Willner et al. 1979 have measured the flux in the $7 \mu \mathrm{m}$ [Ar II] line as $4.9 \times 10^{-11} \mathrm{erg} \mathrm{s}^{-1} \mathrm{~cm}^{-2}$, which yields $n\left(\mathrm{Ar}^{+}\right) / n\left(\mathrm{H}^{+}\right)=1.4 \times$ $10^{-6}$. The neon abundance is increased over earlier estimates. It would appear that theoretical models tend to overestimate $n\left(\mathrm{Ne}^{++}\right) / n\left(\mathrm{Ne}^{+}\right)$, but this point requires further investigation.

NGC 2867: Optical data from Aller et al. 1981.

IC 4593: The optical region data are from Czyzak, Buerger, and Aller 1975. An earlier theoretical model by Buerger 1973 yielded a much smaller argon abundance.

NGC 6210: The optical region data are from Czyzak and Aller 1970; the [S III] data are from Barker 1978.
NGC 6572: A well observed object with a rich spectrum. The optical region spectrum has been interpreted by a detailed model (Aller et al. 1980). Note the corrections to Table V of Czyzak and Aller 1979, $\log I(4959)=2.63 ; \log I(5517)=-0.72$, and $\log I(5755)$ $=0.15$

NGC 6803: The optical data are from Lee et al. 1970.

NGC 6886: The $\lambda 9069$ [S III] data are from Perinotto 1974.

NGC 7009: Czyzak and Aller 1979 analyzed major and minor axes and ansae separately. The nebula shows great differences in excitation along the major and minor axes.

TABLE 4

LOG $N$ FOR NEBULAE

\begin{tabular}{cccc}
\hline \hline Name & $\mathrm{Ne}$ & \multicolumn{1}{c}{$\mathrm{S}$} & $\mathrm{Ar}$ \\
\hline IC $418 \ldots \ldots \ldots$ & $7.88(0.15)^{\mathrm{a}}$ & $6.79(0.12)^{\mathrm{a}}$ & $6.26(0.15)^{\mathrm{a}}$ \\
IC $2165 \ldots \ldots \ldots$ & $7.91(0.15)$ & $6.75(0.12)$ & $6.30(0.12)$ \\
NGC $2867 \ldots \ldots$ & $7.90(0.12)$ & $6.73(0.18)$ & $6.26(0.15)$ \\
NGC $3242 \ldots \ldots$ & $7.85(0.12)$ & $6.69(0.15)$ & $6.26(0.15)$ \\
IC $4593 \ldots \ldots \ldots$ & $7.53(0.12)$ & $6.78(0.18)$ & $6.40(0.15)$ \\
NGC $6210 \ldots \ldots$ & $7.98(0.12)$ & $7.35(0.15)$ & $6.30(0.15)$ \\
Me $2-1 \ldots \ldots \ldots$ & $8.18(0.15)$ & $7.16(0.12)$ & $6.41(0.12)$ \\
NGC $6572 \ldots \ldots$ & $8.00(0.12)$ & $6.68(0.18)$ & $6.40(0.12)$ \\
NGC $6741 \ldots \ldots$ & $8.23(0.15)$ & $6.92(0.18)$ & $6.70(0.15)$ \\
NGC $6790 \ldots \ldots$ & $7.79(0.12)$ & $6.85(0.15)$ & $6.20(0.15)$ \\
NGC $6803 \ldots \ldots$ & $8.38(0.12)$ & $7.12(0.18)$ & $6.76(0.15)$ \\
NGC $6884 \ldots \ldots$ & $7.98(0.12)$ & $7.05(0.12)$ & $6.58(0.15)$ \\
NGC $6886 \ldots \ldots$ & $8.21(0.15)$ & $7.24(0.12)$ & $6.45(0.15)$ \\
NGC $7026 \ldots \ldots$ & $8.26(0.12)$ & $7.61(0.15)$ & $6.64(0.15)$ \\
IC $5117 \ldots \ldots \ldots$ & $7.97(0.12)$ & $7.08(0.12)$ & $6.38(0.15)$ \\
IC $5217 \ldots \ldots \ldots$ & $7.92(0.12)$ & $7.24(0.12)$ & $6.36(0.15)$ \\
NGC $7662 \ldots \ldots$ & $7.82(0.15)$ & $6.83(0.15)$ & $6.32(0.15)$ \\
& & & \\
log $\langle N\rangle \ldots \ldots$. & 8.03 & 7.08 & 6.44 \\
Aller $(1978 b) \ldots$ & 8.02 & 6.97 & 6.38 \\
\hline
\end{tabular}

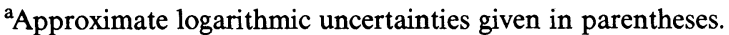


large. In truncated models, $r_{c}$ is less than $r_{\mathrm{s}}$. At each radius the computer solves for the ionization equilibrium of the various elements. The requirement of energy balance fixes $T_{e}$. Physical processes involved include collisional excitation of low-lying levels of abundant elements, photoionization and recombination (including dielectric recombination), and charge exchange. Reaction rates for the latter are often highly uncertain.

To calculate a model network, the procedure has been to choose initially a standard chemical composition based on previous work. Stellar fluxes were taken from calculations by Cassinelli (1971) for effective temperatures of 37,496, 48,865 , and $95,090 \mathrm{~K}$ and for a modified blackbody flux of about $T=80,000 \mathrm{~K}$. We chose only a single sequence of stellar radii ranging from 1.0 solar radius to 0.3 solar radius for the hottest star. For each model five different values of $r_{c}$ were selected.

From the diagnostics of any nebular spectrum, one can deduce $N_{e}, T_{e}$, and ratios such as $n\left(\mathrm{He}^{+2}\right) / n\left(\mathrm{He}^{+}\right)$. Also we can tabulate such excitation dependent ratios as $I(5007) / I(3727)$, i.e., [O $\mathrm{III}] /[\mathrm{O} \mathrm{II}]$ for low to medium excitation objects and $I(3426) / I(3868)$, i.e., $[\mathrm{Ne} \mathrm{V}] /[\mathrm{Ne} \mathrm{III}]$ for high excitation nebulae. Necessary data for most of the objects are given by Aller and Czyzak (1979).

The network of theoretical models provided a framework within which interpolation could be made to fit the general level of excitation of any particular nebula. A fine tuning of the initial models permitted an improved representation of the line intensities for most objects, often by adjusting $F^{*}(\nu)$. For a few objects we relied on interpolated models. IC 4997 was a notable problem. Diagnostic data suggested that the observed spectrum came from strata over a very large temperature and density range. No satisfactory theoretical model for this nebula could be found, nor was an adequate model found for the highly stratified nebula NGC 7009. We have therefore excluded IC 4997 from the analysis and excluded NGC 7009 from the sample used to calculate mean abundances for all modeled nebulae.

It should be emphasized that theoretical models have limitations aside from those imposed by geometrical irregularities, condensations, etc. One example is carbon in IC 418. Observations by Harrington et al. (1980) give $n\left(\mathrm{C}^{+}\right) / n\left(\mathrm{C}^{+2}\right) \sim 3.0$, while their theoretical models, as well as those by Flower (1969) and the present models, predict $n\left(\mathrm{C}^{+}\right) / n\left(\mathrm{C}^{+2}\right)=0.5$. Harrington et al. $(1980)$ suggest that charge exchange may account for the discordance. Butler, Bender, and Dalgarno (1979), however, find no support for a large charge exchange rate. The problem is difficult and confusing.

Another example is [Ar IV]. If the intensities of the [Ar III] lines are fitted to the model, the theoretical [Ar IV] intensities are far too strong for many nebulae. Here the difficulty may be in the atomic parameters for $\mathrm{Ar}^{+3}$ (Czyzak et al. 1980).

The uncertainties in abundances produced by modeling are difficult to estimate but are thought to be roughly $\pm 20 \%$. This degree of uncertainty does not include the uncertainties in atomic constants and rate coefficients. Fortunately, for most ions in most of the nebulae considered here, the ICFs do not differ very much from 1, which means that most of the ionic states are observed.

\section{b) Equations for Calculation of Ionic Abundances}

The ground electron configuration and ground term are $2 p^{5}$ and ${ }^{2} P$ for $[\mathrm{Ne} \mathrm{II}], 3 p^{1}$ and ${ }^{2} P$ for [S IV], and $3 p^{4}$ and ${ }^{3} P$ for [Ar III]. The infrared lines involve transitions between the fine-structure levels of the ground terms of those ions.

The fine-structure emission line intensities can be calculated from the equations of Aller and Liller (1968) or Petrosian (1970). The ionic abundances are then given by:

$$
\begin{aligned}
& \frac{N\left(\mathrm{Ne}^{+}\right)}{N\left(\mathrm{H}^{+}\right)}=8.106 \times 10^{-5}\left(1+\frac{x}{37.2}\right) t^{1 / 2}{E_{4,2}^{0}}(t) 10^{0.0489 / t} \frac{I(12.8 \mu \mathrm{m})}{I\left(\mathrm{H}_{\beta}\right)} \\
& \frac{N\left(\mathrm{~S}^{+3}\right)}{N\left(\mathrm{H}^{+}\right)}=6.31 \times 10^{-6}\left(1+\frac{x}{7.70}\right) t^{1 / 2}{E_{4,2}^{0}}^{0}(t) 10^{0.0595 / t} \frac{I(10.5 \mu \mathrm{m})}{I\left(\mathrm{H}_{\beta}\right)} \\
& \frac{N\left(\mathrm{Ar}^{+2}\right)}{N\left(\mathrm{H}^{+}\right)} \approx 9.58 \times 10^{-6}\left(1+\frac{x}{20}\right) t^{1 / 2} E_{4,2}^{0}(t) 10^{0.0695 / t} \frac{I(9.0 \mu \mathrm{m})}{I\left(\mathrm{H}_{\beta}\right)}
\end{aligned}
$$

Where $x=n_{e} / 10^{4} \mathrm{~cm}^{-3}, t=T_{e} / 10^{4} \mathrm{~K}$, and $E_{4,2}^{0}(t) \approx 1$ has been tabulated by Clark (see Aller and Liller 1968). Collision strengths are taken from Seaton (1975), Brocklehurst (1971), and Krueger and Czyzak (1970). In these equations, the collisional de-excitation terms,

$$
\left(1+\frac{x}{x_{\text {crit }}}\right)
$$


have been calculated according to the method of Petrosian (1970), which corrects for the population of the excited level which is normally neglected in optical transitions. The collisional de-excitation term for [Ar III] is only approximate, as it does not correct exactly for the population of the third level of the ${ }^{3} P$ term, but the correction is always small ( $\left.\lesssim 4 \%\right)$ for the nebulae studied here.

In the optical and near-infrared ranges, ionized $\mathrm{Ne}, \mathrm{S}$, and $\mathrm{Ar}$ are revealed by lines of [Ne III], [Ne IV], [Ne v], [S II], [S III], [Ar III], [Ar IV], and [Ar v]. Ionic concentrations may be calculated from these forbidden line intensities when the atomic parameters are known. For example, $N\left(\mathrm{Ar}^{+2}\right) / N\left(\mathrm{H}^{+}\right)$may be computed from $\lambda 7135$ by an expression of the form (Czyzak and Aller 1981)

$$
\frac{N\left(\mathrm{Ar}^{+2}\right)}{N\left(\mathrm{H}^{+}\right)}=9.221 \times 10^{-7}(1+0.0022 x) t^{1 / 2} E_{4,2}^{0}(t) 10^{0.841 / t} \frac{I(7135)}{I(\mathrm{H} \beta)},
$$

where the atomic parameters are taken from Czyzak and Krueger (1963) and Krueger and Czyzak (1970), except that the collision strengths have been reassessed.

The ionic concentration, $n\left(\mathrm{Ar}^{+3}\right) / n\left(\mathrm{H}^{+}\right)$, is calculated from the nebular-type transition $\lambda 4740$. It may be uncertain since the use of collision strengths published by Saraph and Seaton (1970) or by Krueger and Czyzak (1970) lead to predicted line intensities that do not fit the observations (Czyzak et al. 1980). $N\left(\mathrm{Ar}^{+4}\right) / n\left(\mathrm{H}^{+}\right)$can be found from [Ar v] $\lambda 6435, \lambda 7005$ nebular-type transitions in high excitation planetaries.

The $n\left(\mathrm{Ne}^{+2}\right) / n\left(\mathrm{H}^{+}\right)$ratio is found from $I(3868) / I(\mathrm{H} \beta)$ which was observed in all of the planetaries. We employ Seaton's (1975) collision strengths. In high excitation planetaries, the auroral transitions of [Ne IV] appear near $\lambda 4725$. The ratio $n\left(\mathrm{Ne}^{+3}\right) / n\left(\mathrm{H}^{+}\right)$may be calculated from $I(4724,4725) / I(\mathrm{H} \beta)$ with the aid of expressions based on solutions of the equation of statistical equilibrium (Aller 1970). Unfortunately, the Boltzmann factor is sensitive to uncertainties in $T_{e}$ which has to be estimated from theoretical models. Also, new collision strengths are needed for [Ne IV]. For $n\left(\mathrm{Ne}^{+4}\right) / n\left(\mathrm{H}^{+}\right)$we employ the [Ne v] nebular-type transitions with collision strengths due to Giles (1979). For neon in high excitation objects, it would appear that the largest uncertainty comes from errors in $T_{e}$.

Usually, $n\left(\mathrm{~S}^{+}\right) / n\left(\mathrm{H}^{+}\right)$is small; it is calculated from the intensities of $\lambda 6717,6730$, using Pradhan's (1978) cross sections. The $n\left(\mathrm{~S}^{+2}\right) / n\left(\mathrm{H}^{+}\right)$ratio is found from $I(9069)$ whenever possible. Otherwise, $\lambda 6312$ has to be used.

\section{DISCUSSION}

The derived ionic concentrations are listed in Table 3 for the 18 nebulae for which we have the most complete data. Only these nebulae are included in the discussion below. For each element, $\mathrm{Ne}, \mathrm{Ar}$, and $\mathrm{S}$, the concentrations of the observed ions (as ratios to $\mathrm{H}^{+}$), the sum of the observed ionic concentrations, the ionization correction factor deduced from theoretical models, and finally the derived abundances are given.

For $\mathrm{Ar}^{+2}$ the optically derived values are listed below the infrared values. In most cases where both are given the agreement is adequate or good and the mean is used in determining the total abundance of argon. The agreement suggests that errors in the atomic parameters for the two transitions either are small or cancel each other. In IC 4593 and NGC 6790 the $\mathrm{Ar}^{+2}$ concentration deduced from the $9 \mu \mathrm{m}$ line is substantially greater than that found from $\lambda 7135$. One probable cause of these discrepancies is variation in ionic abundance across the nebulae combined with the differences in beam sizes used for the infrared and optical measurements. Errors in electron temperature and internal extinction at $\lambda 7135$ may also contribute in these particular nebulae, although the generally good agreement between infrared and optically derived $\mathrm{Ar}^{+2}$ abundances suggest that they are usually not excessive. The measurement of $\lambda 7135$ in IC 4593 could have been hampered by scattered light from the central star. In calculating the elemental abundance of argon in these two nebulae the infrared measurement of $\mathrm{Ar}^{+2}$ has been used.

Table 4 gives the finally adopted $\log N$ 's for 17 nebulae. The logarithms of the mean elemental abundances of this group of nebulae are given at the bottom of Table 4 and are compared with the values found by Aller $(1978 b)$. Rough uncertainties in the $\log N$ for each element in each nebula are also given. These vary because of (1) different uncertainties in the infrared and optical measurements, (2) whether the dominant ionic species is determined from infrared or optical measurements, and (3) whether one or more ionic species dominates. A summary of the progression of errors, from observation to elemental abundance determination, is given in Table 5.

Several of the planetary nebulae in Table 4 have abundances which differ by a factor of 2 or more from the mean nebular values. NGC 7026 appears enriched in all three elements, most prominently in sulfur, although the large [S IV] flux determined here may be in disagreement with previous observations. NGC 6803 appears enriched in neon and argon. NGC 6741 is enhanced in argon, but NGC 6790 is deficient in this element (note that this abundance determination uses the $\mathrm{Ar}^{+2}$ abundance derived from the infrared line, which is much larger than the optically derived value). NGC 6572 and NGC 3242 are deficient in sulfur, while NGC 6210 is enhanced (the large [S IV] flux reported 
TABLE 5

SUMMARY OF UNCERTAINTIES

\begin{tabular}{lccccc}
\hline \hline & $\begin{array}{c}\text { Measured } \\
\text { Flux }\end{array}$ & $\begin{array}{c}\text { Total } \\
\text { Flux } \\
\text { Correction }\end{array}$ & Model & $\begin{array}{c}\text { Ionic } \\
\text { Abundance }\end{array}$ & $\begin{array}{r}\text { Elemental } \\
\text { Abundance }^{\mathrm{a}}\end{array}$ \\
\hline Optical & $15 \%$ & $15 \%, 25 \% \mathrm{~b}$ & $20 \%$ & $30 \%, 40 \% \mathrm{~b}$ & $30 \%-60 \%$ \\
Infrared & $15 \%$ & $0-25 \%$ & $20 \%$ & $25 \%-40 \%$ & \\
\hline
\end{tabular}

${ }^{\mathrm{a}}$ See text, § IV.

${ }^{\mathrm{b}}$ For transauroral [S III] $\lambda 6312$.

here for NGC 6210 is in some disagreement with previously reported values, however). IC 4593 shows a large neon underabundance; however, it is somewhat surprising that the [Ne II] line was not detected in this low excitation nebula.

The logarithms of the mean abundances of neon, sulfur, and argon in these 17 planetary nebulae are 8.03, 7.08, and 6.44 respectively. The agreement of the neon abundance with Aller's (1978b) study is close, but the newly derived sulfur and argon abundances are enhanced compared to his values by $30 \%$ and $15 \%$, respectively. Some of this difference can be attributed to the use of improved models. From 12 planetary nebulae, Dinerstein (1980) found $\log \langle N(\mathrm{~S})\rangle=7.32$ as compared with the present value of 7.08 , but if the same collision strengths are used, the difference is much reduced.

Planetary nebulae are generally regarded as having originated from stars of 2-5 solar masses. In more massive objects, nuclear processes have modified the concentration of elements such as nitrogen, as is evident for such nebulae as NGC 6302 and 6886 (Aller and Keyes 1981). More massive stars are required to produce $\mathrm{Ne}, \mathrm{Mg}$, Si, etc., which is returned eventually to the interstellar medium. Thus the abundances of elements such as $\mathrm{Ne}, \mathrm{S}$, and $\mathrm{Ar}$ in planetary nebulae should reflect the composition of the material from which the progenitor stars were formed. In the simplest scenario of element building, we might expect the ratio $\mathrm{Ne}: \mathrm{S}: \mathrm{Ar}$ to be roughly constant from one object to another.

According to the above, it is reasonable that none of the planetary nebulae in Table 4 show combinations of large overabundances and underabundances in the three elements studied. Consider now the more detailed correlation of $\log N(\mathrm{Ne})$ with $\log N(\mathrm{Ar})$. Except for IC 4593, most of the abundances fit a $45^{\circ}$ correlation line to within a factor of 2 . Per $\log$ of argon or neon abundance, however, $\log N(\mathrm{~S})$ shows a much larger spread and cannot be reconciled with a $45^{\circ}$ slope. For example, if the S/Ar ratio in NGC 2165, NGC 3242, or IC 418 is taken as "normal," NGC 6210, NGC 6886, NGC 7026, and IC 5217 all show a large relative excess of sulfur. Perhaps S is tied up in grains with an efficiency that varies with the type of nebula.

The acquisition and reduction of optical observations was supported in part by NSF grant 77-21022 to UCLA. The infrared measurements were supported in part by NASA grant 05-003-272 and by NSF grant AST 78-24453. We wish to thank the staffs of Lick Observatory and Las Campanas Observatory for their assistance with the observations. S. C. B. thanks the Institute of Theoretical Physics at the University of California at Santa Barbara for its hospitality.

\section{REFERENCES}

Aitken, D. K., Roche, P. F., Spenser, P. M., and Jones, B. 1979, Ap. J., 233, 925.

Aller, L. H. 1970, Proc. Nat. Acad. Sci., 65, 775. 1976, Pub. A. S. P., 88, 574. 1978 , in Planetary Nebulae, ed. Y. Terzian (Dordrecht: Reidel), p. 225 $1978 b$, Proc. Astron. Soc. Australia, 3, 213.

Aller, L. H., and Czyzak, S. J. 1979, Ap. Space Sci., $62,397$.

Aller, L. H., and Keyes, C. D. 1981, "First Two Years of the IUE," in NASA Symposium, in press.

Aller, L. H., Keyes, C. D., O'Mara, B. J., and Ross, J. E. 1981, M.N.R.A.S., in press.

Aller, L. H., Keyes, C. D., Ross, J. E., and Czyzak, S. J. 1980, Ap. Space Sci., 67, 349.

Aller, L. H., and Liller, W. 1968, in Stars and Stellar Systems, ed. B. Middlehurst and L. H. Aller (Chicago: University of Chicago Press), 7, 483.

Barker, T. 1978, $A p . J ., 221,145$.

Brocklehurst, M. 1971, M.N.R.A.S., 153, 471

Buerger, E. 1973, Ap. J., 160, 887.
Butler, S. E., Bender, C. F., and Dalgarno, A. 1979, Ap. J. (Letters), 230, L59.

Cassinelli, J. P. 1971, Ap. J., 165, 265.

Czyzak, S. J., and Aller, L. H. 1970, Ap. J., 162, 783.

Czyzak, S. J., and Aller, L. H. 1979, M.N.R.A.S., 188, 229. 1981 , in preparation.

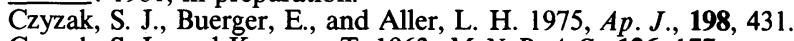
Czyzak, S. J., and Krueger, T. 1963, M.N.R.A.S., 126, 177.

Czyzak, S. J., Sonneborn, G., Aller, L. H., and Shectman, S. A. 1980, Ap. J., 241, 719.

Dinerstein, H. 1980, Ap. J., 237, 486.

Flower, D. R. 1969, M.N.R.A.S., 146, 171.

Giles, K. 1979, M.N.R.A.S., 180, 57P.

Gillett, F. C., Merrill, M., and Stein, W. A. 1972, Ap. J., 172, 367.

Grandi, S. A. and Hawley, S. A. 1978, Pub. A. S. P., 90, 125.

Grasdalen, G. 1979, Ap. J., 229, 587.

Harrington, J. P., Lutz, J. H., Seaton, M. J., and Stickland, D. J. 1980, M.N.R.A.S., 191, 13.

Kaler, J. B. 1976, Ap. J. Suppl., 31, 517.

Keyes, C. D. and Aller, L. H. 1979, Ap. Space Sci., 59, 91. 
Krueger,T., and Czyzak, S. J. 1970, Proc. Roy. Soc. London, A, 318, 531 .

Lacy, J. H. 1979, Ph.D. thesis, University of California, Berkeley. Lee, P., Aller, L. H., Kaler, J. B., and Czyzak, S. J. 1974, Ap. J., $192,159$.

Liller, W., and Aller, L. H. 1963, Proc. Nat. Acad. Sci., 49, 675

Milne, D., and Aller, L. H. 1975, Astr. Ap., 38, 183.

O'Dell, C. R. 1962, Ap. J., 135, 371.

O'Dell, C. R., and Terzian, Y. 1970, Ap. J., 160, 915.

Peimbert, M., and Costero, R. 1969, Bol. Obs. Tonanzintla y Tecubaya, 5, 3.
Perinotto, H. 1974, Astr. Ap., 35, 293.

Petrosian, W. 1970, Ap. J., 159, 833.

Pradhan, A. K. 1978, M.N.R.A.S., 184, 89P

Rank, D. 1978, in Planetary Nebulae, ed. Y. Terzian (Dordrecht: Reidel), p. 103.

Saraph, H. E., and Seaton, M. J. 1970, M.N.R.A.S., 148, 367.

Seaton, M. J. 1975, M.N.R.A.S., 170, 475.

Willner, S. P., Jones, B., Puetter, R. C., Russell, R. W., and Soifer, B. T. 1979, Ap. J., 234, 496.

L. H. Aller: Astronomy Department, University of California, Math Sciences Building, Los Angeles, CA 90024

F. BAAS: Huygens Laboratorium, Rijksuniversiteit, P.O. Box 9504, 2300 RA Leiden, The Netherlands

S. C. BECK: Department of Physics, University of California, Berkeley, CA 94720

T. R. Geballe: Mount Wilson and Las Campanas Observatories, 813 Santa Barbara Street, Pasadena, CA 91101

J. H. LACY: Physics Department, California Institute of Technology, 320-47, Pasadena, CA 91125

C. H. Townes: Department of Physics, University of California, Berkeley, CA 94720 